\title{
ESTRATIFICAÇÃO E DENSIDADE DE INVERTEBRADOS EM SOLO ARENOSO SOB FLORESTA PRIMÁRIA E PLANTIOS ARBÓREOS NA AMAZÔNIA CENTRAL DURANTE A ESTAÇÃO SECA.
}

\section{Ana Yoshi HARADA ${ }^{1.2}$, Adelmar Gomes BANDEIRA ${ }^{2,3}$}

\begin{abstract}
RESUMO - Densidade e distribuição vertical de invertebrados foram estudados durante o mês de setembro de 1990 e 1991, em solo de três tipos de coobertura vegetal; floresta primária, plantios de Dipteryx odorata (Leguminosae = cumaru) e Simaruba amara $($ Simarubaceae $=$ marupá), nos arredores de Manaus, Brasil. Foram encontrados 19 grupos principais da fauna. A maioria desses grupos foram similares para os três tipos de cobertura, sendo Isoptera $(40,1 \%)$, Formicidae $(25,2 \%)$, Araneae $(6,7 \%)$ os grupos mais abundantes que, juntos, representaram $62,4 \%$ da fauna de cumaru, $57,0 \%$ da fauna de marupá e $84,1 \%$ da fauna de floresta. Houve uma diminuição da densidade para todos os grupos (excluídos os cupins) até a profundidade de $20 \mathrm{~cm}$, com subsequiente aumento até $30 \mathrm{~cm}$ de profundidade, principalmente pela presença de Isoptera. Cerca de $45 \%$ de toda a fauna ocorreu no estrato de $0-5 \mathrm{~cm}$ de profundidade, seguido de $9,5 \%$ (5-10 $\mathrm{cm}), 9,1 \%(10-15 \mathrm{~cm}), 2,7 \%(15-20 \mathrm{~cm}), 20,7 \%(20-25 \mathrm{~cm})$ e $12,3 \%(25-30 \mathrm{~cm})$ nos estratos subseqüentes. Isoptera, Diptera e outros invertebrados não apresentaram correlação com a umidade e o $\mathrm{pH}$ do solo.
\end{abstract}

Palavras-chave: Invertebrados do solo, densidade, distribuição vertical, solo arenoso, estação seca, floresta, plantio arbóreo, Amazônia Central.

Stratification and Density of Invertebrates from Sandy Soil under Forest and two Arboreal Plantations in Central Amazonia, During the Dry Season.

\begin{abstract}
Density and vertical distribution of soil invertebrates were studied during September of 1990 and 1991 in three plots, namely one forest plot and two arboreal plantations (Dipteryx odorata = cumaru <Leguminosae> and Simaruba amara = marupá < Simarubaceae >) near Manaus (Brazil). Nineteen main fauna groups were found, and most of them had similar densities in the three plots. The most representative groups were Isoptera $(40.1 \%)$, Formicidae $(25.2 \%)$, Araneae $(6.7 \%)$, all together representing $62.4 \%$ of all soil fauna in the cumaru plot, $57.0 \%$ in manupa and $84.1 \%$ in the forest plot. Density of soil fauna decreased from the top soil to $20 \mathrm{~cm}$ depth (excluding termites), with a subsequent increase until $30 \mathrm{~cm}$ depth. $45 \%$ of all fauna occurred in the top $0-5 \mathrm{~cm}$, followed by $9.5 \%(5-10 \mathrm{~cm}), 9.1 \%(10-15$ $\mathrm{cm}), 2.7 \%(15-20 \mathrm{~cm}), 20.7 \%(20-25 \mathrm{~cm})$ and $12.3 \%(25-30 \mathrm{~cm})$. Isoptera, Diptera and other invertebrates did not present correlation with the $\mathrm{pH}$ and the humidity of the soil.
\end{abstract}

Key-words: Soil invertebrates, density, vertical distribution, sandy soil, dry season, forest, arboreal plantaion, Central Amazonia.

\section{INTRODUÇÃO}

O fluxo de energia no meio edáfico em grande parte é mantido pela atividade da fauna do solo, que tem importante papel nos processos de decomposição da matéria orgânica, nos ciclos de nutrientes, na aeração e

I Instituto Nacional de Pesquisas da Amazônia, Coordenadoria de Pesquisas em Entomologia, Caixa Postal 478, 69.011-970 Manaus, AM, Brasil.

2 Bolsista do CNPq.

3. Universidade Federal da Paraíba, Departamento de Sistemática e Ecologia, CCEN, 58.059900 João Pessoa, PB, Brasil. 
fertilidade do solo, entre outras funções (EDWARDS et al., 1970; SERAFINO \& MERINO, 1978; CHAUVEL, et al., 1987). Já existem trabalhos de pesquisa mostrando que na Amazônia Central as comunidades de invertebrados do solo em pastagens, onde antes era floresta, têm a densidade grandemente reduzida e a composição dos grupos muito diferente daquela do ambiente primário (DANTAS, 1978; BANDEIRA \& TORRES, 1985). Essas alterações parecem ser menos severas quando o solo é utilizado para culturas arbóreas (BANDEIRA \& SOUZA, 1983; MELO, 1984).

No presente trabalho mostra-se um estudo comparativo da macrofauna em solo arenoso sob monoculturas arbóreas de Dipteryx odorata $($ Leguminosae $=$ cumaru) e Simaruba amara (Simarubaceae = marupá) em relação à floresta, com o objetivo de identificar os grupos de invertebrados presentes, sua densidade em diferentes estratos até $30 \mathrm{~cm}$ de profundidades, abundância relativa e o efeito que a umidade e $\mathrm{o} \mathrm{pH}$ podem ter sobre a densidade.

\section{ÁREA DE ESTUDO}

A pesquisa foi realizada na área de terra firme denominada $\mathrm{S} 1 \mathrm{da}$ Estação Experimental de Silvicultura Tropical do INPA, km 45 da BR 174 , Manaus-Boa Vista $\left(2^{\circ} 35^{\prime} \mathrm{S} ; 60^{\circ} \mathrm{W}\right)$. Nesta região da Amazônia Central, próximo de Manaus, o total médio de precipitação anual é de $2.105 \mathrm{~mm}$, tendo uma estação chuvosa de dezembro a maio com $75 \%$ das chuvas e uma estação menos chuvosa de junho a novembro com $25 \%$ das chuvas registradas (RIBEIRO \& SANTOS, 1975; RIBEIRO \& ADIS, 1984). Em torno de $70 \%$ dos solos de terra firme (áreas não alagáveis) são latossolos amarelos, de textura arenosa a muito argilosa (FALESI, 1967; FALESI \& SILVA, 1969; PENNY \& ARIAS, 1982), Foram selecionadas três coberturas vegetais, sendo floresta primária e duas áreas de experimentos florestais arbóreos com 18 a 19 anos de idade (implantadas em 1972).

A floresta era uma transição entre campinarana e floresta sensu PIRES (1974) e BRAGA (1979), com as árvores maiores estimadas em $30 \mathrm{a}$ $35 \mathrm{~m}$ de altura; seu sub-bosque era relativamente limpo, permitindo bom trânsito de pessoas.

Os experimentos florestais estudados foram um plantio de cumaru (Dipteryx odorata) e um de marupá (Simaruba amara) ambos com espaçamento de $5 \times 5 \mathrm{~m}$. As árvores de cumaru atingiam aproximadamente de 12-15 $\mathrm{m}$ de altura e as de marupá tinham em torno de $20 \mathrm{~m}$ de altura. As copas das árvores de cumaru eram mais abertas e continham mais folhas (maior sombreamento) que as de marupá, sendo que esta última cultura permitia a passagem de maior quantidade de radiação solar. A quantidade de folhas e de galhos caídos (liteira) era maior sobre o solo do plantio de manupá. Talvez por ser mais iluminado, o sub-bosque sob marupá era mais desenvolvido que o de cumaru, inclusive com predominância de Pterydophyta.

Cumaru e marupá são essências 
florestais nativas da Amazônia. A madeira de cumaru é bastante durável e é muito utlizada em construções residenciais; seus frutos, oleosos e de odor forte, servem para fabricação de sabão, medicamento caseiro e outras finalidades. Marupá, cuja madeira é leve e pouco resistente, tem sido muito utilizada na fabricação de caixotes e palitos de fósforo.

Para melhor caracterização da área, foi feita análise granulométrica do solo, de amostras compostas de 10 unidades, coletadas de $0-30 \mathrm{~cm}$ de profundidade sob cada uma das três coberturas vegetais. $\mathrm{O}$ método utilizado foi o da pipeta (CAMARGO et al., 1986). Na floresta, encontrou-se $75 \%$ de areia, $6 \%$ de silte e $18 \%$ de argila (franco arenoso); no plantio de cumaru, encontrou-se $92 \%$ de areia, $3 \%$ de silte e $5 \%$ de argila (areia); no plantio de marupá, encontrou-se $79 \%$ de areia, $16 \%$ de silte e $5 \%$ de argila (areia franca). Entretanto, não fez parte do escopo deste trabalho comparar a abundância da fauna com a granulometria dos estratos do solo.

Mais informações sobre o solo de uma área bem próxima a esta, incluindo dados de morfologia e gênese, são encontrados em CHAUVEL et al. (1987).

\section{METODOLOGIA}

Sob cada cobertura vegetal foi demarcada uma parcela de $300 \mathrm{~m}^{2}$, estabelecidos 31 pontos, distanciados $5 \mathrm{~m}$ entre si, que foram sorteados ao acaso para a retirada de amostras de solo para extração da macrofauna. Em duas estações de estiagem, mês de setembro de 1990 e de 1991, foram retiradas 10 amostras (blocos) de solo (cinco por estação) de $20 \times 20 \times$ $30 \mathrm{~cm}$ em cada uma das três parcelas.
Cada amostra de solo foi seccionada em estratos de $5 \mathrm{~cm}$ (uma amostra dando seis subamostras de $20 \times 20 \times 5 \mathrm{~cm})$. ANDERSON \& INGRAM (1989) recomendam, para pesquisas em áreas tropicais, de cinco a 10 amostras de solo de $25 \times 25 \times 30 \mathrm{~cm}$ por estação, seccionadas a cada $10 \mathrm{~cm}$ de altura (cada amostra dando três subamostras de $25 \times 25 \times 10 \mathrm{~cm}$ ). Entretanto, na Região Amazônica, o solo em sua maioria é muito argiloso, compacto e/ou contém muitas raízes nesta parte superficial, o que dificulta muito a retirada de amostras maiores que as acima referidas, principalmente em áreas de floresta primária, mesmo que em solo arenoso. Todas as coletas foram feitas entre 7:00 e 9:00 horas para efeito de comparação.

Os animais foram separados manualmente logo após a retirada do solo, ainda no campo, e acondicionados em vidros contendo álcool a $75 \%$. A identificação dos animais foi feita nos níveis de classe, ordem (a maioria dos grupos) e família (Formicidae). Devido à baixa densidade e freqüência dos invertebrados, os insetos das ordens Dermaptera, Embioptera, Homoptera, Hymenoptera (Vespoidea), Lepidoptera (imaturos), Neuroptera, Thysanoptera e Zoraptera foram agrupadas como Outros Insetos; Crustacea, Pauropoda e Nematoda foram agrupados como Outros Invertebrados, e Opiliones, Palpigradi e Ricinulei como outros Arachnida (Tab.1 e Tab. 2).

Distribuição vertical dos invertebrados em relação às condições abióticas da profundidade do solo foram analisadas com o teste de 
correlação linear e teste $\mathbf{t}$ de Student, usando os pacotes estatísticos SAS/ STAT v. 6.03, Systat v.5.02 (WILKINSON, 1990) e Statgraph v. 5.0 (POLHEMUS, 1988). Os dados originais foram transformados em $\sqrt{x+0.5}$, para dar maior uniformidade, e foram utilizados nas análises (ZAR, 1974). Ass variáveis foram cobertura vegetal (cumaru, marupá e floresta), número de indivíduos da fauna, $\mathrm{pH}$ e umidade do solo. $\mathrm{O} \mathrm{pH}$ do solo foi determinado em água (CAMARGO $\mathrm{et}$ al., 1986), usando pH-metro CORNING, modelo 7. As determinações foram feitas com parte do solo retirado para extração dos animais, de estratos de $5 \mathrm{~cm}$ de espessura, de $0-30 \mathrm{~cm}$ de profundidade, cada amostra com três repetições, para estimativa das médias.

A umidade do solo foi obtida pelo método gravimétrico (ALLEN, 1974), calculada pela fórmula: Umidade do solo $=$ peso do solo úmido - peso do solo seco $\times 100$ / peso do solo úmido.

Todo material ficará depositado na Coleção de Invertebrados do INPA.

\section{RESULTADOS}

Encontraram-se 19 grupos de invertebrados (após a junção dos grupos muito pouco expressivos), no total de 2.330 indivíduos, cuja representação de cada grupo (abundância [números originais], dominância [percentuais] e densidade [indivíduos $/ \mathrm{m}^{2}$ ]), distribuição por estrato e por cobertura vegetal são mostrados nas Tab. 1 e Tab. 2. Isoptera, Formicidae e Araneae foram os grupos mais abundantes nos três tipos de coberturas vegetais, juntos representando
$62,4 \%$ do total da fauna no cumaru, $57,0 \%$ no marupá e $84,1 \%$ na floresta.

Não houve padronização na distribuição vertical da fauna entre as três parcelas, sendo que a maior abundância de indivíduos ocorreu de $0-5 \mathrm{~cm}$ de profundidade no cumaru (372 indivíduos) e no marupá (393 indi-víduos) e de 20-25 $\mathrm{cm}$ de profundidade na floresta (447 indivíduos) (influenciada por Isoptera), e a menor abundância de $15-20 \mathrm{~cm}$ nas três parcelas $(\mathrm{Cum}=11$ indivíduos; $\mathrm{Mar}=3$ indivíduos e $\mathrm{Flo}=50$ indivíduos), cujos dados são mostrados juntos com os de umidade e de $\mathrm{pH}$ (Tab.2, Fig.1, Fig.2). Formicidae, Araneae, Chilopoda e Coleoptera imaturo diminuíram sua dominância do estrato superficial até o estrato de $15-20 \mathrm{~cm}$, a partir do qual houve um aumento até a profundidade de $30 \mathrm{~cm}$ (Tab.1, Fig.3). Entretanto, Isoptera foi dominante em relação aos demais grupos no estrato de $20-25 \mathrm{~cm}$ de profundidade, chegando a influenciar no total da fauna (Fig.1, Fig.2, Fig.3).

A abundância da maioria dos grupos da fauna mostrou correlação positiva e significativa a nível de 5\% com a umidade do solo (Tab. 3). Uma das poucas exceções foi Isoptera, um dos grupos mais abundantes (principalmente na floresta), que foi negativa em duas das parcelas, mas não significativa a nível de $5 \%$. A umidade do solo variou de 12,6 a 31,5 na floresta, de 12,9 a 22,0 no marupá e de 7,7 a 14,1 no cumaru (Fig. 1).

O número de indivíduos, quando correlacionado com os valores de $\mathrm{pH}$ do solo dos plantios florestais, foi negativo $\mathrm{e}$ significativo a nível de $5 \%$ para a maioria dos grupos (Tab.3). Ao contrário de todos 
Tabela 1. Dominância (\%) de invertebrados do solo, coletados durante a estação menos chuvosa. em 10 amostras de solo arenoso de $20 \mathrm{~cm} \times 20$ por $30 \mathrm{~cm}$ de profundidade, divididas em estratos de $5 \mathrm{~cm}(0-5,5-10$ 10-15, 10-15, 15-20, 20-25, 25-30) em três parcelas, da área S1 da Estação Experimental de Silvicultura Tropical do INPA, região de Manaus, Amazônia Central. (Cum=Cumaru, Dipleryx odorata; Mar=Marupá, Simaruba amara; e Flo=Floresta; números entre perênteses representam totais de indivíduos; $X=$ médias e $\mathrm{DP}=$ desvios padröes; zeros=sem registros).

\begin{tabular}{|c|c|c|c|c|c|c|c|c|c|}
\hline \multirow{2}{*}{$\begin{array}{l}\text { GRUPOS DA } \\
\text { FAUNA }\end{array}$} & \multicolumn{9}{|c|}{ PROFUNDIDADES (CM) } \\
\hline & & $0-5$ & $5-10$ & $10-1$ & $15-2$ & $20-2$ & $25-3$ & $0-30$ & $\mathrm{X} \pm \mathrm{DP}$ \\
\hline & Cum & 2,6 & 0,0 & 0,0 & 0,0 & 0,0 & 0,0 & $2,6(15)$ & $2,5 \pm 6,1$ \\
\hline \multirow[t]{3}{*}{ Collembola } & Mar & 3,3 & 0,0 & 0,0 & 0,0 & 0,0 & 0,0 & $3,3(20)$ & $3,3 \pm 8,2$ \\
\hline & Flo & 0,3 & 0,0 & 0,0 & 0,0 & 0,0 & 0,0 & $0,3(03)$ & $0,5 \pm 1,2$ \\
\hline & Cum & 3,3 & 0,7 & 0,0 & 0,2 & 0,2 & 0,0 & $4,4(25)$ & $4,2 \pm 7,4$ \\
\hline \multirow[t]{3}{*}{ Diplura } & Mar & 2,5 & 0,2 & 0,3 & 0,0 & 0,0 & 0,2 & $3,2(19)$ & $3,2 \pm 5,8$ \\
\hline & Flo & 3,1 & 0,5 & 0,1 & 0,1 & 0,0 & 0,0 & $3,8(44)$ & $7,3 \pm 14,2$ \\
\hline & Cum & 2,6 & 0,0 & 0,0 & 0,0 & 0,2 & 0,2 & $3,0(17)$ & $2,8 \pm 6,0$ \\
\hline \multirow[t]{3}{*}{ Coleoptera adulto } & Mar & 7,2 & 0,2 & 0,0 & 0,0 & 0,0 & 0,2 & $7,6(45)$ & $7,5 \pm 17,4$ \\
\hline & Flo & 1,8 & 0,0 & 0,0 & 0,1 & 0,1 & 0,0 & $2,0(23)$ & $3,8 \pm 8,5$ \\
\hline & Cum & 0,4 & 0,0 & 0,0 & 0,0 & 0,0 & 0,0 & $0,4(02)$ & $0,3 \pm 0,8$ \\
\hline \multirow[t]{3}{*}{ Coleoptera imaturo } & Mar & 0,0 & 0,0 & 0,0 & 0,0 & 0,0 & 0,0 & $0,0(00)$ & $0,0 \pm 0,0$ \\
\hline & Flo & 0,2 & 0,3 & 0,0 & 0,0 & 0,1 & 0,0 & $0,6(06)$ & $1,0 \pm 1,3$ \\
\hline & Cum & 0,4 & 0,0 & 0,0 & 0,0 & 0,2 & 0,0 & $0,6(03)$ & $0,5 \pm 0,8$ \\
\hline \multirow[t]{3}{*}{ Diptera } & Mar & 0,2 & 0,0 & 0,0 & 0,0 & 0,0 & 0,0 & $0,2(0,1)$ & $0,5 \pm 0,4$ \\
\hline & Flo & 0,1 & 0,2 & 0,0 & 0,0 & 0,0 & 0,0 & $0,3(03)$ & $0,5 \pm 0,8$ \\
\hline & Cum & 0,2 & 0,0 & 0,0 & 0,0 & 0,0 & 0,0 & $0,2(01)$ & $0,2 \pm 0,4$ \\
\hline \multirow[t]{3}{*}{ Hemiptero } & Mar & 0,7 & 0,0 & 0,0 & 0,0 & 0,0 & 0,0 & $0.7(04)$ & $0,7 \pm 1,6$ \\
\hline & Flo & 0,2 & 0,1 & 0,0 & 0,0 & 0,0 & 0,0 & $0,3(03)$ & $0,5 \pm 0,8$ \\
\hline & Cum & 20,8 & 6,6 & 2,1 & 1,1 & 2,6 & 2,5 & $35,7(204)$ & $34,0 \pm 43,1$ \\
\hline \multirow[t]{3}{*}{ Hym. (Formicidae) } & Mar & 18,9 & 5,0 & 4,4 & 0,3 & 1,7 & 0,5 & $30,7(184)$ & $30,7 \pm 42,0$ \\
\hline & Flo & 9,1 & 2,2 & 1,6 & 1,4 & 0,9 & 1,7 & $17,0(195)$ & $32,5 \pm 35,9$ \\
\hline & Cum & 0,9 & 1,2 & 2,3 & 0,2 & 0,9 & 11,0 & $16,5(94)$ & $15,7 \pm 23,5$ \\
\hline \multirow[t]{3}{*}{ Isoptera } & Mar & 0,0 & 0,2 & 16,6 & 0,2 & 0,3 & 0,3 & $17,6(105)$ & $17,5 \pm 39,9$ \\
\hline & Flo & 0,7 & 5,2 & 2,7 & 2,5 & 37,4 & 14,9 & $63,4(735)$ & $122,5 \pm 163,5$ \\
\hline & Cum & 4,0 & 0,2 & 0,2 & 0,0 & 0,0 & 0,0 & $4,4(25)$ & $4,2 \pm 9,2$ \\
\hline \multirow[t]{3}{*}{ Orthoptera } & Mar & 1,5 & 0,3 & 0,0 & 0,0 & 0,0 & 0,0 & $1,8(11)$ & $1,8 \pm 3,6$ \\
\hline & Flo & 0,2 & 0,0 & 0,0 & 0,0 & 0,0 & 0,0 & $0,2(02)$ & $0,3 \pm 0,8$ \\
\hline & Cum & 0,4 & 0,0 & 0,0 & 0,0 & 0,0 & 0,0 & $0,4(02)$ & $0,3 \pm 0,8$ \\
\hline \multirow[t]{2}{*}{ Outros Insetos } & Mar & 0,3 & 0,2 & 0,0 & 0,0 & 0,0 & 0,0 & $0,5(03)$ & $0,5 \pm 0,8$ \\
\hline & Flo & 0,8 & 0,1 & 0,0 & 0,0 & 0,1 & 0,0 & $1,0(11)$ & $1,8 \pm 3,5$ \\
\hline \multicolumn{10}{|l|}{ ARACHNIDA } \\
\hline & Cum & 0,4 & 0,0 & 0,0 & 0,0 & 0,0 & 0,0 & $0,4(02)$ & $0,3 \pm 0,8$ \\
\hline \multirow[t]{3}{*}{ Acarina } & Mar & 2,0 & 0,0 & 0,0 & 0,0 & 0,0 & 0,0 & $2,0(12)$ & $2,0 \pm 4,9$ \\
\hline & Flo & 0,3 & 0,0 & 0,0 & 0,0 & 0,0 & 0,0 & $0,3(04)$ & $0,7 \pm 1,6$ \\
\hline & Cum & 10,0 & 0,0 & 0,2 & 0,0 & 0,0 & 0,2 & $10,4(59)$ & $9,8 \pm 23,1$ \\
\hline \multirow[t]{3}{*}{ Araneae } & Mar & 8,0 & 0,0 & 0,2 & 0,0 & 0,0 & 0,2 & $8,7(52)$ & $8,7 \pm 19,3$ \\
\hline & Flo & 3,4 & 0,3 & 0,0 & 0,2 & 0,0 & 0,1 & $4,0(46)$ & $7,7 \pm 15,4$ \\
\hline & Cum & 1,2 & 0,0 & 0,0 & 0,0 & 0,0 & 0,0 & $1,2(07)$ & $1,2 \pm 2,9$ \\
\hline \multirow[t]{2}{*}{ Pseudoescorpionida } & Mar & 3,2 & 0,0 & 0,0 & 0,0 & 0,0 & 0,0 & $3,2(19)$ & $3,2 \pm 7,8$ \\
\hline & Fio & 0,6 & 0,1 & 0,0 & 0,0 & 0,0 & 0,0 & $0,7(08)$ & $1,3 \pm 2,8$ \\
\hline
\end{tabular}


continuaçāo tabela 1.

\begin{tabular}{|c|c|c|c|c|c|c|c|c|c|}
\hline \multirow{3}{*}{ Outros Arachnida } & Cum & 1,2 & 0,0 & 0,0 & 0,0 & 0,0 & 0,0 & $1,2(07)$ & $1,2 \pm 2,9$ \\
\hline & Mar & 2,0 & 0,0 & 0,0 & 0,0 & 0,0 & 0,0 & $2,0(12)$ & $2,0 \pm 4,9$ \\
\hline \multirow{2}{*}{\multicolumn{10}{|c|}{$\begin{array}{c}\text { OUTROS } \\
\text { ARTHROPODA }\end{array}$}} \\
\hline & & & & & & & & & \\
\hline & Cum & 2,5 & 0,4 & 0,0 & 0,0 & 0,2 & 0,0 & $2,9(17)$ & $2,8 \pm 5,5$ \\
\hline \multirow[t]{3}{*}{ Chilopoda } & Mar & 2,8 & 0,8 & 0,0 & 0,0 & 0,0 & 0,0 & $3,6(22)$ & $3,7 \pm 6,8$ \\
\hline & Flo & 0,8 & 0,3 & 0,0 & 0,0 & 0,0 & 0,1 & $1,2(14)$ & $2,3 \pm 3,6$ \\
\hline & Cum & 4 & 0,2 & 0,0 & 0,0 & 0,0 & 0,0 & $4,2(27)$ & $4,0 \pm 9,3$ \\
\hline \multirow[t]{3}{*}{ Diplopoda } & Mar & 4,2 & 0,0 & 0,3 & 0,0 & 0,0 & 0,0 & $4,5(27)$ & $4,5 \pm 10,1$ \\
\hline & Flo & 1,0 & 0,1 & 0,0 & 0,0 & 0,0 & 0,0 & $1,1(12)$ & $2,0 \pm 4,4$ \\
\hline & Cum & 6,3 & 0,0 & 0,0 & 0,0 & 0,0 & 0,0 & $6,3(36)$ & $6,0 \pm 14,7$ \\
\hline \multirow[t]{3}{*}{ Isopoda } & Mar & 4,2 & 0,0 & 0,0 & 0,0 & 0,0 & 0,0 & $4,2(25)$ & $4,2 \pm 10,2$ \\
\hline & Flo & 1,3 & 0,0 & 0,0 & 0,1 & 0,0 & 0,0 & $1,4(16)$ & $2,7 \pm 6,1$ \\
\hline & Cum & 1,9 & 0,0 & 0,0 & 0,0 & 0,0 & 0,0 & $1,9(11)$ & $1,8 \pm 4,5$ \\
\hline \multirow[t]{3}{*}{ Symphyla } & Mar & 1,8 & 0,0 & 0,0 & 0,0 & 0,0 & 0,0 & $1,8(11)$ & $1,8 \pm 4,5$ \\
\hline & Fla & 0,1 & 0,1 & 0,0 & 0,0 & 0,0 & 0,0 & $0,2(02)$ & $0,3 \pm 0,5$ \\
\hline & Cum & 2,1 & 0,5 & 0,4 & 0,0 & 0,0 & 0,0 & $3,4(19)$ & $3,2 \pm 4,5$ \\
\hline \multirow[t]{3}{*}{ Oligochaeta } & Mar & 1,2 & 1,5 & 0,0 & 0,0 & 0,0 & 0,0 & $2,7(16)$ & $2,7 \pm 4,2$ \\
\hline & Flo & 1,2 & 0,3 & 0,2 & 0,0 & 0,0 & 0,0 & $1,7(19)$ & $3,2 \pm 5,5$ \\
\hline & Cum & 0,0 & 0,0 & 0,0 & 0,0 & 0,0 & 0,4 & $0,4(02)$ & $0,3 \pm 0,8$ \\
\hline \multirow[t]{3}{*}{ Outros Invertebrados } & Mar & 0,0 & 0,0 & 0,0 & 0,0 & 0,0 & 0,0 & $0,0(00)$ & $0,0 \pm 0,0$ \\
\hline & Flo & 0,0 & 0,0 & 0,0 & 0,0 & 0,0 & 0,2 & $0,2(02)$ & $0,3 \pm 0,8$ \\
\hline & Cum & 65,0 & 9,8 & 5,1 & 1,8 & 4,2 & 14,2 & $100(572)$ & $95,3 \pm 137,9$ \\
\hline \multirow[t]{2}{*}{ Total por Parcela } & Flo & 65,7 & 8,7 & 21,7 & 0,5 & 2,0 & 2,3 & $100(598)$ & $99,7 \pm 151,4$ \\
\hline & Mar & 25.8 & 9.8 & 4.6 & 4,3 & 38,5 & 17.0 & $100(1160)$ & $193,3 \pm 155,9$ \\
\hline
\end{tabular}

Obs: os valores $X$ e DP foram obtidos a partir da somatória de invertebrados encontrados nas 10 amostras dos seis estratos $(0-30 \mathrm{~cm}$ de profundidade) em cada parcela de estudo.

Tabela 2. Abundância ( $\mathrm{n}^{\circ}$ de animais) e densidade $\left(\mathrm{n}^{\circ}\right.$ de indivíduos $\left./ \mathrm{m}^{2}\right)$ durante a estação menos chuvosa, em 10 amostras de solo arenoso de $20 \mathrm{~cm} \times 20 \mathrm{~cm} \times 30 \mathrm{~cm}$ de profundidade, divididas em estratos de $5 \mathrm{~cm}(0-5,5-10,10-15,15-20,20-25,25-30)$ em três parcelas da área S1 da Estação Experimental de Silvicultura Tropical do INPA, região de Manaus, Amazônia Central, de 1990-1991.

\begin{tabular}{lccccccc}
\hline VARIÁVEIS/ESTRATO & $0-5$ & $5-10$ & $10-15$ & $15-26$ & $20-25$ & $25-30$ & Total \\
\hline $\begin{array}{l}\text { Abundância } \\
\text { Cumaru }\end{array}$ & 372 & 56 & 29 & 11 & 24 & 81 & 573 \\
$\begin{array}{l}\text { Marupá } \\
\text { Floresta }\end{array}$ & 393 & 52 & 130 & 3 & 12 & 8 & 598 \\
$\begin{array}{l}\text { Densidade } \\
\text { Cumaru }\end{array}$ & 299 & 114 & 53 & 50 & 447 & 107 & 1160 \\
Marupá & 930,0 & 140,0 & 72,5 & 25,0 & 60,0 & 202,5 & 1430 \\
$\begin{array}{l}\text { Floresta } \\
\text { No de Grupos }\end{array}$ & 982,5 & 130,0 & 325,0 & 7,5 & 30,0 & 20,0 & 1495 \\
Cumaru & 747,5 & 285,0 & 132,5 & 125,0 & 1117,5 & 492,5 & 2900 \\
Marupá & 19 & 7 & 5 & 4 & 6 & 6 & 20 \\
Floresta & 17 & 9 & 5 & 2 & 2 & 5 & 20 \\
\hline
\end{tabular}


Tabela 3. Níveis de correlação, durante a estação menos chuvosa, de invertebrados terrestres (número de indivíduos) com umidade e $\mathrm{pH}$ em solo arenoso na área $\mathrm{SI}$ da Estação Experimental de Silvicultura Tropical do INPA, na Amazônia Central $\left(* \mathrm{p}<5 \% ;{ }^{* *} \mathrm{p}<1 \%\right.$; n.s. $=$ não significativo; --. sem registro); rus = umidade do solo; $\mathrm{rph}=\mathrm{pH}$ do solo.

\section{GRUPOS}

DA

FAUNA
Dipteryx odorata

rus

\section{COBERTURA VEGETAL}

Simanuba amara

Floresta

rus $\quad \mathrm{pH}$

\section{INSECTA}

Collembola

$0,966^{\star *} \quad-0,967^{\star \star} \quad 0,962^{\star *} \quad-0,850^{\star} \quad 0,993^{\star \star *} \quad-0,718$ n.s.

Diplura

$0,865^{*} \quad-0,966^{\star *} \quad 0,980^{\star \star *} \quad-0,884^{*} \quad 0,918^{\star *} \quad-0,821^{*}$

Coleoptera adulto

$0,921^{\star *} \quad 0-906 \quad 0,970^{\star *} \quad-0,857^{*} \quad 0,968^{\star \star} \quad-0,731$ n.s.

Coleoptera imaturo

$0,966^{\star *} \quad-0,967^{* *}$

Diptera

0,654 n.s.

$--$

-.. $\quad 0,395$ n.s. $-0,798$ n.s.

Hemiptera

$\begin{array}{llllll}0,966^{\star \star} & -0,967^{\star \star} & 0,962^{\star \star} & -0,850^{*} & 0,806^{*} & -0,884^{\star}\end{array}$

Hym. (Formicidae)

$0,851^{*} \quad-0,958^{*} \quad 0,837^{*}$

$-0,889^{*} \quad 0,964^{* \star} \quad-0,732$ n.s.

Isoptera

Lepidoptera

Orthoptera

$-0,133$ n.s. $-0,231$ n.s. $-0,160$ n.s. 0,107 n.s. 0,426 n.s. $-0,299$ n.s.

Outros insecta

$0,966^{\star \star} \quad-0,967^{\star \star} \quad 0,962^{\star \star} \quad-0,850^{\star} \quad \ldots$

$0,958^{\star \star} \quad-0,988^{\star \star \star} \quad 0,815^{\star} \quad-0,918^{\star \star} \quad 0,993^{\star \star \star} \quad-0,918 n \cdot s$

0,575 n.s. $-0,621$ n.s. 0,560 n.s. $-0,587$ n.s. $0,940^{\star *} \quad-0,772^{*}$

ARACHNIDA

\begin{tabular}{|c|c|c|c|c|c|c|}
\hline Acarina & $0,966^{\star \star}$ & $-0,967^{\star \star}$ & $0,962^{\star *}$ & $-0,850^{*}$ & $0,993^{\star \star \star}$ & $-0,718$ n.s. \\
\hline Araneae & $0,974^{\star \star \star}$ & $-0,957^{\star \star}$ & $0,971^{\star \star}$ & $-0,887^{\star}$ & $0,940^{\star *}$ & $-0,840^{*}$ \\
\hline Pseudoescorpionida & $0,966^{\star \star}$ & $-0,970^{\star \star}$ & $0,962^{\star \star}$ & $-0,850^{*}$ & $0,949^{\star \star}$ & $-0,826^{\star}$ \\
\hline Outros Arachnida & $0,966^{\star *}$ & $-0,967^{\star \star}$ & $0,962^{\star *}$ & $-0,850^{\star}$ & $0,993^{\star \star \star *}$ & $-0,718$ n.s. \\
\hline \multicolumn{7}{|l|}{$\begin{array}{l}\text { OUTROS } \\
\text { ARTHROPODIA }\end{array}$} \\
\hline Chilopoda & $0,887^{\star}$ & $-0,963^{\star \star}$ & $0,830^{*}$ & $-0,915^{\star \star}$ & $0,866^{*}$ & $-0,874^{\star}$ \\
\hline Diplopoda & $0,953^{\star \star}$ & $-0,987^{\star \star \star}$ & $0,966^{\star \star}$ & $-0,864^{*}$ & $0,966^{\star \star}$ & $-0,804^{\star}$ \\
\hline Isopoda & 0.966 & $-0,962^{\star \star}$ & $0,962^{\star \star}$ & $-0,850^{*}$ & $0,965^{\star \star}$ & $-0,730$ n.s. \\
\hline Symphyla & $0,966^{\star \star}$ & $-0,967^{\star *}$ & $0,962^{\star \star}$ & $-0,850^{*}$ & 0,596 n.s. & $-0,854^{*}$ \\
\hline Oligochaeta & 0,261 n.s. & $-0,802^{*}$ & 0,333 n.s. & $-0,805^{*}$ & 0,423 n.s. & $-0,860^{* *}$ \\
\hline Outros invertebrados & $-0,102$ n.s. & $-0,714$ n.s. & -- & -- & 0,517 n.s. & $-0,606$ n.s. \\
\hline TOTAL DE GRUPOS & 0,261 n.s. & $-0,802^{*}$ & $-0,333$ n.s. & $-0,805^{\star}$ & $-0,423$ n.s. & $-0,860^{\star \star}$ \\
\hline
\end{tabular}


Cumaru

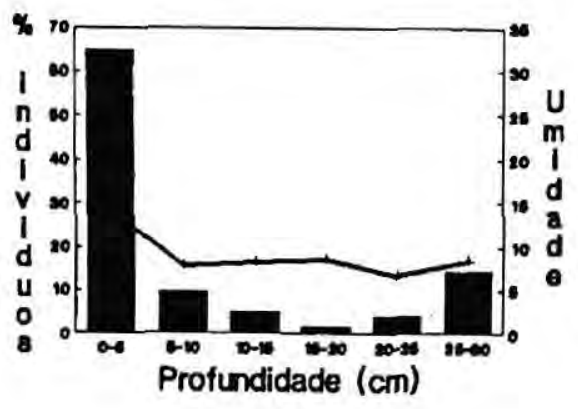

Marupa

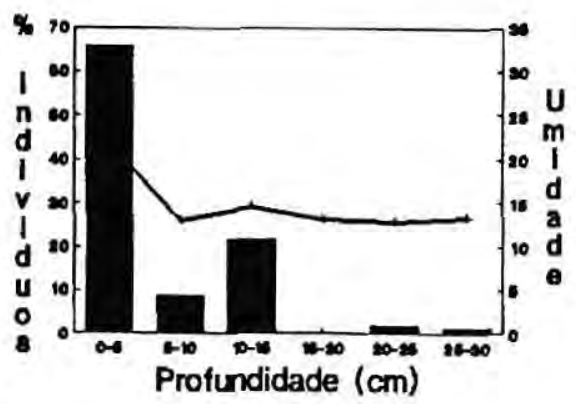

Floresta

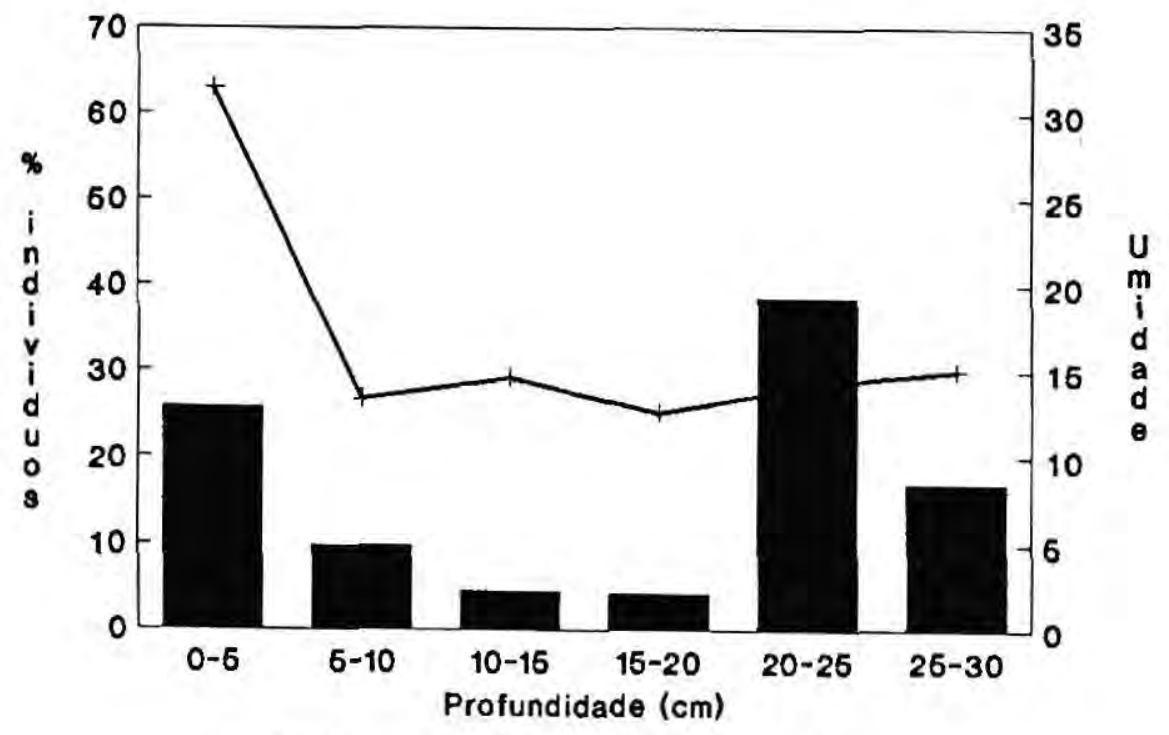

\% individuos $\rightarrow$ umidade solo

Figura 1. Distribuição vertical da fauna e umidade do solo sob três coberturas vegetais na Amazônia Central. 

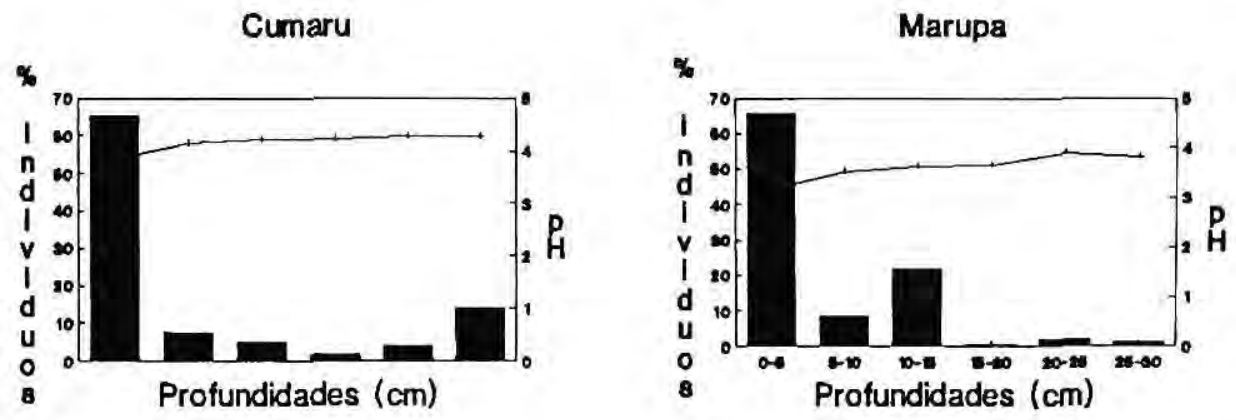

Floresta

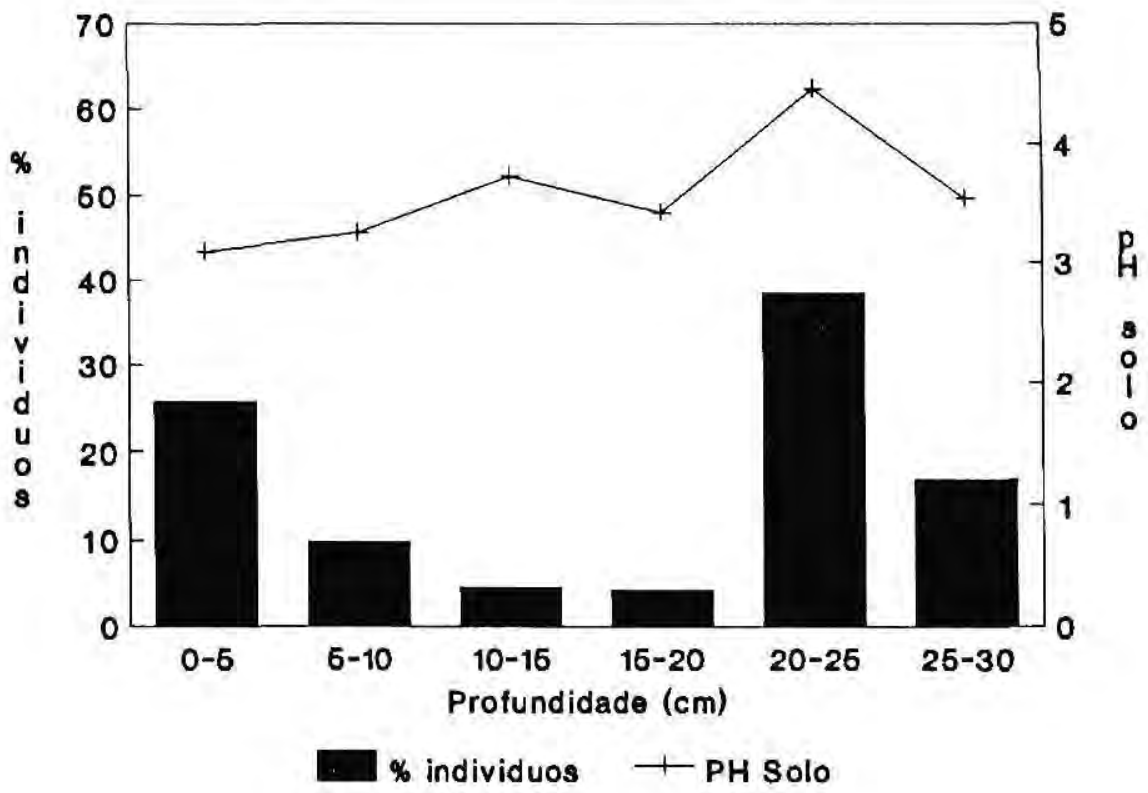

Figura 2. Distribuição vertical da fauna e pH do solo sob três coberturas vegetais na Amazônia central. 


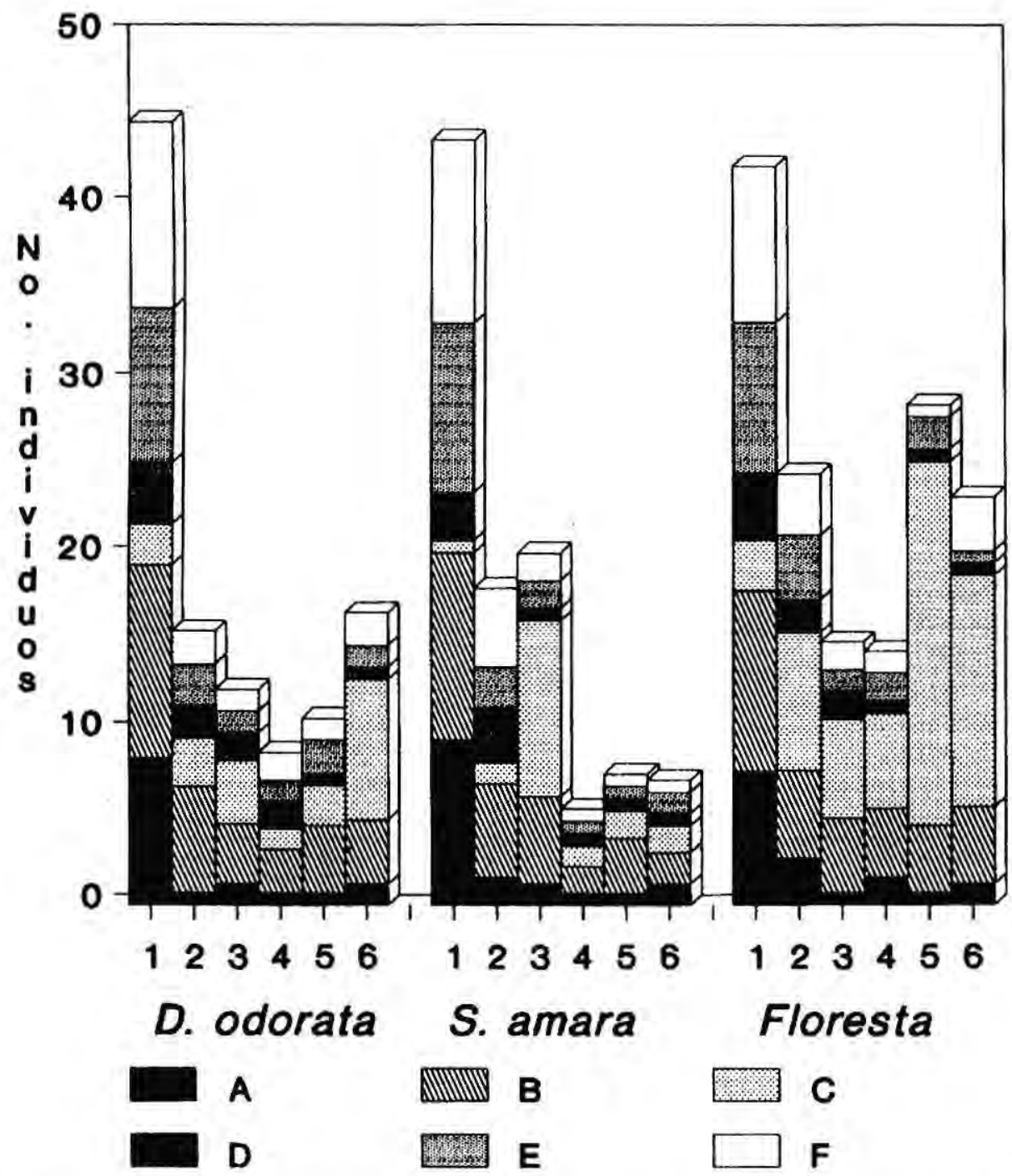

Figura 3. Densidade dos seis principais grupos de invertebrados de solo em seis estratos de $5 \mathrm{~cm}$. indo da superfície até a profundidade de $30 \mathrm{~cm}$, em floresta e plantios de $D$. odorala e $S$. amara. Estratos: I $(0-5 \mathrm{~cm}) ; 2(5-10 \mathrm{~cm}) 3(10-15 \mathrm{~cm}) ; 4(15-20 \mathrm{~cm}) ; 5(20-25 \mathrm{~cm})$ e $6(25-30 \mathrm{~cm})$. $\mathrm{A}=$ Araneae, $\mathrm{B}=$ Formicidae, $\mathrm{C}=$ Isoptera, $\mathrm{D}=$ Oligochaeta, $\mathrm{E}=$ Outros insetos e $\mathrm{F}=$ Outros invertebrados. 
os demais táxons, Isoptera (grupo mais abundante) teve correlação positiva com o $\mathrm{pH}$ em duas das parcelas, mas não significativa a nível de $5 \%$. Isso mostra a resistência dos cupins a valores de $\mathrm{pH}$ do solo bastante ácido, que variou de 3,1 a 3,5 na floresta, 3,2 a 3,8 no marupá e 3,8 a 4,3 no cumaru (Fig.2). Entretanto, tenhamos de convir que correlações a nível de grupos (p. ex. Isoptera, Formicidae, Pseudoescorpionida, etc.) podem camuflar "correlações contrárias" a nível de espécies.

O método de coleta foi bastante eficiente na captura de animais relativamente grandes, como Isoptera (40,1\% da fauna das três parcelas), Formicidae $(25,2 \%)$, Araneae $(6,7 \%)$, Diplura $(3,8 \%)$, Coleoptera adulto $(3,7 \%)$, Isopoda $(3,3 \%)$ e Oligochaeta $(2,3 \%)$, e pouco eficiente na captura da mesofauna, como Collembola $(1,6 \%)$ e Acarina $(0,8 \%)$, muito embora estes dois últimos grupos tenham representado até $80 \%$ de toda a fauna do solo na Região Amazônica, quando coletada por outros métodos (DANTAS, 1978; ADIS, 1989a,b; BANDEIRA \& TORRES, 1985, 1988).

\section{DISCUSSÃO E CONCLUSÕES}

Conhecimentos acerca da fauna de invertebrados do solo na Amazônia (p. ex. DANTAS, 1978; BANDEIRA \& TORRES, 1985; MELO, 1984; MORAIS, 1985; RODRIGUES, 1986) não atingem áreas de plantios florestais estudados neste trabalho. Os resultados desta pesquisa indicam que não houve diferença significativa a nível de $5 \%$ no número de grupos de invertebrados encontrados na floresta em relação àqueles coletados nas duas parcelas de cultivos florestais, durante o período de estiagem.

A densidade de invertebrados foi maior na floresta do que nos experimentos florestais, influenciada principalmente por Isoptera (Tab.2), de forma semelhante ao encontrado por LIEBERMAN \& DOCK (1982) comparando floresta com plantação de cacau; entretanto, estes resultados discordam daqueles de SERAFINO \& MERINO (1978), que encontraram menores densidades nas faunas de floresta e pastagem do que em monoculturas.

Cerca de $65 \%$ dos animais coletados nos plantios de cumaru e marupá e $25 \%$ daqueles na floresta ocupavam o estrato superficial (de 0-5 $\mathrm{cm}$ de profundidade), havendo um decréscimo da densidade até a profundidade de $20 \mathrm{~cm}$, seguido de novo acréscimo até à profundidade de $30 \mathrm{~cm}$, nos três tipos de cobertura vegetal (Fig. 1, Fig. 2 e Fig.3). Pela literatura que se teve acesso, esta é a primeira vez que tal fato é registrado para a Amazônia. A concentração da fauna no estrato superficial ( 0 $5 \mathrm{~cm}$ ) foi maior nos plantios arbóreos que na floresta; o decréscimo da concentração foi no sentido cumaru $(18,6 \pm 27,4)$ marupá $(19,2 \pm 25,8)$ - floresta $(15,0 \pm$ $23,8)$, tomando-se grupos em particular, como Formicidae $(20,8 \%, 18,9 \%$ e $9.1 \%$ ) e Araneae $(9,9 \%, 8,0 \%$ e $3,4 \%$ ); porém, se comparada a fauna de $0-30 \mathrm{~cm}$ de profundidade, a densidade foi maior na floresta (Tab.1 e Tab.2). Resultados semelhantes foram encontrados por RODRIGUES (1986). $\mathrm{O}$ aumento da abundância nos estratos mais profundos, principalmente na floresta, foi devido principalmente à presença de Isoptera. Entretanto, a 
distribuição de Pseudoescorpionida encontrada por ADIS \& MAHNERT (1993) numa floresta primária próxima a nossa área de estudo não concordam com os nossos resultados para esses animais, fato que talvez seja devido a metodologia.

Trabalhos comparando fauna do solo de pastagens com fauna de floresta primária na Amazônia (DANTAS, 1978; BANDEIRA \& TORRES, 1985; MELO, 1984) têm mostrado que não só a densidade é menor em pastagens, mas também o número de grupos tem sido sempre inferior ao encontrado em floresta. Neste trabalho, o número de grupos foi semelhante entre as monoculturas e a floresta; porém ainda não é possível afirmar se esta semelhança na diversidade entre as três parcelas é devida às espécies vegetais que compõem as monoculturas, ou à idade dos plantios (19 anos), ou se foi devido à metodologia (inclusive a profundidade até $30 \mathrm{~cm}$ ), pela primeira vez empregada para este tipo de estudo na Amazônia Brasileira.

Considerando que as perturbações ambientais influenciam as populações de invertebrados do solo, Isoptera foi o único grupo mais abundante na floresta $(62,8 \%)$ do que em áreas manejadas, resultados que corroboram os princípios de Thienemamm (apud TISCHLER, 1955) que diz: "quanto mais variáveis as condições de vida de um biótipo, tanto maior será o número de espécies da biocinese correspondente". Assim, a abundância de Formicidae nos estratos superficiais nas monoculturas talvez seja em decorrência de sua grande tolerância a mudanças ambientais, ou devido às características da liteira das monoculturas estudadas, fato que corrobora as afirmações de LIEBERMAN \& DOCK (1982), LEVINGS \& WINDSOR (1985) e MELO (1984). Destaca-se aínda a presença de Symphyla na floresta, que tinha sido encontrado por MELO (1984) apenas em áreas manejadas.

Fatores importantes que também podem influenciar diretamente as comunidades de invertebrados do solo são biomassa microbiana (STRAALEN et al., 1988), quantidade da liteira produzida (LEVINGS, 1984) e outros fatores (como presas, predadores) que possam afetar a disponibilidade de alimentos (FOSTER, 1982) e estão diretamente associados com a biologia de cada grupo (LEVINGS \& WINDSOR, 1982).

Quando se relacionou umidade do solo com a abundância da fauna em relação à profundidade, verificou-se que apenas Isoptera mostrou correlação negativa nos plantios perenes, sendo não significativa, a nível de 5\%, para todos os locais. A correlação com $\mathrm{pH}$ foi inversa à observada com umidade, mas os cupins continuaram a se comportar de maneira diferente da maioria dos outros grupos. Resultados semelhantes foram encontrados por ADIS et al. (1989b). Como a abundância da fauna diminuiu até aproximadamente $20 \mathrm{~cm}$ de profundidade e aumentou novamente a partir daí, mesmo teoricamente havendo menor quantidade 
de alimentos a essa profundidade, isto sugerem que a umidade e o $\mathrm{pH}$ podem realmente influenciar a distribuição vertical da fauna.

Não há indicação, até o momento, de que haja migração de invertebrados do solo, na Amazônia Central, para as camadas mais profundas em resposta apenas à umidade da superfície, como tem sido mencionado para outras regiões (LIEBERMAN \& DOCK, 1982; PETERSEN \& LUXTON, 1982; WILLIS, 1976). Por outro lado, LEVINGS \& WINDSOR (1985) sugerem que a correlação negativa da fauna de invertebrados com a umidade do solo, em relação à profundidade, está relacionada com a estivação das espécies no período de estiagem.

\section{AGRADECIMENTOS}

À Fundação Banco do Brasil (FBB- processo $n^{\circ} 1591$ ), pelo apoio financeiro; ao Conselho Nacional de Desenvolvimento Científico e Tecnológico (CNPq- processos $\mathrm{n}^{\circ}$ 30.0704-5 e 501889/91-4), pela concessão de bolsas de pesquisa; a Noeli Paulo Fernandes e servidores de apoio da Estação Experimental de Silvicultura Tropical (EEST) pelas facilidades para os trabalhos de campo; a José Edvaldo Chaves e Eliana Tamar Ribeiro pela ajuda nas análises de solo e $\mathrm{pH}$; a Eliana Tamar Ribeiro pela triagem da fauna, a Alice dos Santos, Edson Palheta e Cláudio Sena, pelo apoio técnico no campo e no laboratório; a Suely Costa e José Camilo Hurtabo Guerrero, pela ajuda nas análises estatísticas; a Cláudio
Ruy V. Fonseca, Lucille M. K. Antony e Flávio J. Luizão pelas críticas e sugestões.

\section{Bibliografia Citada}

ADIS, J. \& V. MAHNERT. 1993. Vertical distribution of pseudoscorpions (Arachnida) in the soil of two different Neotropical primary forests during the dry and rainy seasons. Mamories of the Queensland Museum 33(2):431-440.

ADIS, J.; MORAIS, J. W.; RIBEIRO, E. F. \& RIBEIRO, J. C. 1989a. Vertical distribution and abundance of arthropods from white sand soil of a Neotropical Campinarana forest during the rainy season. Studies on Neotropical Fauna Environment 24(4):193-200.

ADIS, J.; RIBEIRO, E. F.; MORAIS, J. W. \& CAVALCANTE, E. T. S. 1989b. Vertical distribution and abundance of arthropods from white sand soil of a Neotropical Campinarana forest during the dry season. Studies on Neotropical Fauna Environment 24(4):201-211.

ADIS,J. \& MAHNERT, V. 1993. Vertical distribution and abundance of Pseudoscorpions (Arachnida) in the soil os two different neotropical primary forests during the dry and rainy seasons. Memories of the Queensland Museum 33(2):431-440.

ALLEN, S, E. 1974. Chemical analysis of ecological materials. Oxford, Blackwell Sci. Publ., Seção 1, p: 21-22.

ANDERSON, J. M. \& INGRAM, J. S. I. 1989. Tropical soil biology and fertility: $a$ handbook of methods. Oxon, C.A.B. International, $171 \mathrm{p}$.

BANDEIRA, A. G. \& SOUZA, P. C. 1983. Influência do pinheiro (Pinus caribea) sobre a fauna do solo na Amazonia. Boletim Museu Paraense Emílio Goeldi, n. série (Zoologia) 114:1-13.

BANDEIRA, A. G. \& TORRES, M. F. P. 1985. Abundância e distribuição de invertebrados do solo em ecossitemas da Amazônia Oriental. O papel ecológico dos cupins. Boletim Museu Paraense Emílio Goeldi, série Zoologia, Belém, 2(1):13-38. 
BANDEIRA, A. G. \& TORRES, M. F. P. 1988. Consideraçōes sobre densidade, abundância e variedades de invertebrados terrestres em áreas florestais de Carajás, sudeste da Amazônia. Boletim Museu Paraense Emílio Goeldi, série Zoologia, 4(2):191-199.

BRAGA, P. I. 1979. Subdivisão fitogeográfica, tipos de vegetação, conservação e inventário florístico da floresta Amazônica. Acta Amazonica 9(4):53-80.

CAMARGO, O. A.; MUNIZ, A. C.; JORGE, J. A. \& VALADARES, J. M. A. S. 1986. Métodos de análise química, mineralógica $e$ física de solos do Instituto Agronômico de Campinas. Boletim Técnico do Instituto Agronômico 106:1-94.

CHAUVEL, A.; GUILLAUMET, J. L. \& SCHUBART, H. O. R. 1987. Importance et distribution des racines et des vivants dans un "latossol" argileux sous forêt amazonienne. Revista du Écologie et Biologie du Sol 24(1):19-48.

CHAUVEL, A.; LUCAS, Y. \& BOULET, R. 1987. The dinamics of the Amazonian forest: On the genesis of the soil mantle of the region of Manaus, Central Amazônia, Brazil. Experientia 43: 234-241.

DANTAS, M. 1978. Pastagens da Amázônia Central: Ecologia e fauna do solo. Acta Amazonica 9(2):1-54 (suplemento).

EDWARDS, C. A.; REICHLE, D. E. \& CONSLEY, D. A. 1970. The role of soil invertebrates in remover of coarse malter and nutrients. In: D. E. Reichle (ed.). Analysis of temperate forest ecossystems. New York, Berlin-Heidelberg, $\mathrm{p}: 167-172$.

FALESI, 1. C. 1967. O estado atual dos conhecimentos sobre os solos da Amazônia brasileira. Acta do Simpósio sobre a Biota Amazônica 1:151-168.

FALESI, I. \& SILVA, B. N. R. 1969. Os solos $d a$ área Manaus -ftacoatiara. Série Estudos e Ensaios. $\mathrm{N}^{\circ}$ 1. Secretaria de Produção do Amazonas, IPEAN, Belém, Pará.

FOSTER, R. B. 1982. Famine on Barro Colorado Island. In: LEIGH, E. G; RAND, A. S. \& WINDSOR, D. M. (eds.). The ecology of a tropical long term rhythms. Washington, D. C., Smithsonian Institution Press, p: 201-
211.

LEVINGS, S. C. 1984, Litter moisture content as a determinant of litter arthropod distribution and abundance during the dry season on Barto Colorado Island, Panama. Biotropica 16(2):125-131.

LEVINGS, S. C. \& WINDSOR, D. M. 1985. Litter arthropod populations in a tropical deciduos forest: relationships between years and arthropod groups. Joumal Animal Ecology 54:61-69.

LIEBERMAN, S. \& DOCK, C. F. 1982. Analysis of the leaf litter arthropod fauna of a lowland tropical evegreen forest site (La Selva, Costa Rica). Revista Biologia Tropical 30:27-34.

MELO, L. A. S. 1984. Impacto do manejo de ecossistemas sobre a mesofauna do solo, em áreas de terra firme, na região de Manaus. Dissertação de Mestrado, Instituto Nacional de Pesquisas da Amazônia e Fundação Universidade do Amazonas, Manaus. $117 \mathrm{p}$.

MORAIS, J. W. de. 1985. Abundância e distribuiçāo vertical de Arthropoda do solo numa floresta primária não inundada. Dissertação de Mestrado, Instituto Nacional de Pesquisas da Amazônia e Fundaçāo Universidade do Amazonas, Manaus, Brasil, $92 \mathrm{p}$.

PENNY, N. D. \& AंRIAS, J. R. 1982. Insects of an Amazon forest. New York, Columbia University Press. 269 p.

PETERSEN, H. \& m. LUXTON. 1982. A comparative analysis of soil fauna populations and their role in decomposition processes. Oikos 39:288-387.

PIRES, J. M. 1974, Tipos de vegetação da Amazônia. Brasil Florestal 17(5):48-58.

POLHEMUS, N. 1988. Statgraphs: STSC. INC. $600 \mathrm{p}$.

RIBEIRO, M. N. G. \& J. ADIS, 1984. Local rainfall variability - a potencial bias for bioecological studies in the Central Amazon. Acta Amazonica 14:159-174.

RIBEIRO, M. N. G. \& A. SANTOS. 1975. Observaçōes microclimáticas no ecossitema Campina Amazônica. Acta Amazonica 
5:183-189.

RODRIGUES, J, M. G. 1986. Abundância $e$ distribuição vertical de artrópodos de solo em capoeira de terra firme. Dissertação de Mestrado. Instituto Nacional de Pesquisas da Amazônia e Fundação Universidade do Amazonas, Manaus, $80 \mathrm{p}$.

SERAFINO, A. \& J. F. MERINO. 1978. Poblaciones de microartrópodos en diferentes suelos de Costa Rica. Revista de Biolgia Tropical 26(1):139-151.

STRAALEN, N. M. van; KRAAK, M. H. S. \& DENNEMAN, C. A. J. 1988. Soil microarthropods as indicators of soil acidification and forest decline in the Veluwe area, The Netherlands. Pedobiologia 32:47-55.
TISCHLER, W. 1955. Effects of agricultural pratice on the soil fauna. In: D. K. Kevan, MCE (ed.). Soil Zoology. London, Buteherworth. p:215-231.

WILKINSON, L. 1990. Systat: The system for Statistics. Evanston. II. Systat. INC. $823 \mathrm{p}$.

WILLIS, E. O. 1976. Seasonal changes in the invertebrate litter fauna on Barro Colorado Island, Panamá. Revista Brasileira de Biologia 36:643-657.

ZAR, J. H. 1974. Biostatistical Analysis. London, Printice- Hall, Inc. 620 p. 